\title{
Tissue resident regulatory T cells: novel therapeutic targets for human disease
}

\begin{abstract}
Xiaohui Zhou ${ }^{1,2 \dagger}$, Jiayou Tang ${ }^{3 \dagger}$, Hao Cao ${ }^{3}$, Huimin Fan $^{1,2,3 \star}$ and Bin $\mathrm{Li}^{4 \star}$
Over the past decade, the ability of regulatory T cells (Tregs) to suppress multiple types of immune cells has received tremendous attention. Mounting evidence has revealed that tissue resident Tregs control non-immunological processes of their target tissues and contribute to a plethora of human diseases. The identification of novel tissue-specific Tregs has highlighted their heterogeneity and complexity. This review summarizes the recent findings for visceral adipose tissue CD4+Foxp3+ regulatory T cells (VAT Tregs), muscle Tregs, bone Tregs and skin memory Tregs, with a focus on their unique functions in local tissues. This interpretation of the roles of tissue-specific Tregs and of their involvement in disease progression provides new insight into the discovery of potential therapeutic targets of human diseases.
\end{abstract} Cellular \& Molecular Immunology (2015) 12, 543-552; doi:10.1038/cmi.2015.23; published online 20 April 2015

Keywords: Regulatory T cells; Tissue Tregs; VAT Tregs; Muscle Repair; Areg; Tumor; Bone; Skin memory Tregs

\section{INTRODUCTION}

Regulatory T cells (Tregs) are characterized by the expression of the transcription factor Foxp3 and have been acknowledged as a key regulator involved in mediating the homeostasis of peripheral tolerance. ${ }^{1}$ Although Tregs account for less than $10 \%$ of peripheral CD4 $+\mathrm{T}$ cells, they have received a tremendous amount of attention due to their functions in suppressing multiple types of immune cells and blocking excessive immune responses, inflammation and tissue destruction. ${ }^{2}$ Moreover, recent studies have reported that tissue resident Tregs can control non-immunological processes. These new findings highlight the importance of tissue-specific Tregs in human diseases.

\section{Characterization of Regulatory $\mathrm{T}$ Cells}

Mouse Tregs were initially discovered as CD4+CD25+ cells by Shimon Sakaguchi in $1995,{ }^{3}$ and their human counterparts were firstly characterized as CD4+CD25+ T cells in $2001 .^{4-7}$ Subsequent studies identified Foxp3 as a master transcription factor and functional marker for mouse Tregs. ${ }^{8}$ However, human Foxp3 is only transiently expressed by activated $\mathrm{T}$ cells, ${ }^{9}$ resulting in the phenotypical and functional heterogeneity of human Tregs. ${ }^{10}$ CD127 was reported to distinguish human Foxp3+ regulatory from activated $\mathrm{T}$ cells; this marker is highly enriched on activated $\mathrm{T}$ cells, but almost undetectable on Tregs. ${ }^{11}$ Furthermore, hypomethylation of the Foxp3 promoter contributes to the expression of Foxp3, making this epigenetic profile a distinguishing criteria between Tregs and activated $\mathrm{T}$ cells. ${ }^{12}$

Foxp3 + Tregs were classified into two main categories: $n$ Tregs (thymus-derived natural Tregs) and iTregs (induced in the periphery from naïve $\mathrm{CD} 4+\mathrm{T}$ cells). ${ }^{13}$ Tregs further demonstrated plasticity under specific circumstances. For example, both nTreg and iTregs can convert to Th17 cells in the presence of IL-6, TGF- $\beta$ or the combination of IL-1 and IL-23. ${ }^{14-21}$ Moreover, nTregs can display a Th1 phenotype and produce IFN- $\gamma$ in the presence of IL- 6 and IL-1 $\beta .^{20,21}$ However, other authors have reported the resistance of iTregs induced by IL- 2 and TGF- $\beta$ to Th17 conversion in the presence of IL- $6 .{ }^{16}$ Another group showed that Treg cells from the inflamed central nervous system (CNS-Treg cells) resisted the conversion to IL-17 production when exposed to IL-6 during experimental allergic encephalitis (EAE) in contrast to their splenic counterparts. These contradictions further illustrate the heterogeneity and distinct stages of Foxp3+ cells. All of these characteristics should be considered in Treg-related immunotherapy.

\section{Suppressive Function of Tregs}

Multiple mechanisms have been proposed for the suppressive functions of Tregs, including suppression by inhibitory

\footnotetext{
${ }^{1}$ Research Center for Translational Medicine, Shanghai East Hospital, Tongji University School of Medicine; ${ }^{2}$ Shanghai Heart Failure Research Center; ${ }^{3}$ Department of Heart Failure, Shanghai East Hospital, Tongji University School of Medicine and ${ }^{4}$ Key Laboratory of Molecular Virology \& Immunology, Unit of Molecular Immunology, Institute Pasteur of Shanghai, Shanghai Institutes for Biological Sciences, Shanghai, 200120, China

*Correspondence to Huimin Fan E-mail: frankfan@tongji.edu.cn or Bin Li, E-mail: binli@sibs.ac.cn

'Dr. Zhou and Dr. Tang contribute equally to this work.

Received: 9 November 2014; Revised: 25 February 2015; Accepted: 25 February 2015
} 
cytokines, cytolysis, metabolic disruption, and modulation of dendritic cells (DCs) maturation or function. ${ }^{22}$

Soluble mediators produced by Tregs, including IL-10, TGF- $\beta$, IL-35 and immunosuppressive metabolites such as adenosine, are responsible for the suppressive function of Tregs. ${ }^{22,23}$ Recent findings that VEGF and amphiregulin (Areg) were produced or overexpressed by tissue resident Tregs added to the panel of paracrine mechanisms of Tregs function. ${ }^{24-26}$ Tregs may also exert their activities by lysing effector cells through the secretion of granzymes A and B and perforin. ${ }^{27,28}$ Moreover, Tregs can disrupt the metabolism of effector cells through IL-2 deprivation. ${ }^{29} \mathrm{nTregs}$ also use cell-cell contact mechanisms to exert their activities. Evidences have suggested that Tregs may inhibit DCs maturation through the interaction of CTLA- 4 and CD80/86 on DCs, thereby suppressing the activation of effector T cells. ${ }^{30,31}$ In the absence of APCs, human Tregs may also regulate the functions of $\mathrm{T}$ effectors via a direct cell contact mechanism in vitro. ${ }^{11}$ Although in vitro and in vivo mouse studies have implicated several mechanisms in tregs activity, the precise molecular mechanisms involved in suppression by human Tregs remain to be determined.

\section{Targets of Tregs}

The targets of Tregs include conventional CD4 $+\mathrm{T}$ helper cells, antibody-producing B cells, DCs, CD8 + cytotoxic T cells, macrophages and neutrophils. Recently, studies have revealed that Tregs may also control non-immunological processes, thereby expanding the scope of Tregs functions. For example, Tregs directly inhibit bone erosion and control bone mass in arthritic mice and nonarthritic wild-type (WT) mice, ${ }^{32}$ visceral adipose resident Tregs regulate metabolic systems $^{33,34}$ and muscle Tregs potentiate muscle repair via the expression of the growth factor Areg. ${ }^{26}$ Moreover, tumor Tregs can produce VEGF, which promotes angiogenesis in the tumor vasculature. ${ }^{24}$ The role of these novel subsets sheds light on promising opportunities to target disease-specific Tregs.

\section{Diverse Populations of Tregs in Tissues}

The presence of diverse subsets of Tregs in tissues other than lymphoid organs in both mice and humans has attracted a great deal of attention over the past few years. ${ }^{26}$ Extensive studies have indicated that Tregs are localized in healthy tissues, inflammatory sites, tumors and immuno-privileged tissues, including skin, lung, adipose tissue, joints, muscles and placenta. Each population may possess unique tissue-specific characteristics in terms of phenotype and function compared to their counterparts in lymphoid organs. ${ }^{25,27,34-40}$ Recently, several populations of tissue resident Tregs were identified, including special Tregs in visceral adipose tissue, ${ }^{33,34}$ muscle Tregs ${ }^{26}$ and skin-resident memory Tregs. ${ }^{41}$ These discoveries broadened the realms of the Tregs family and highlighted some insightful biological implications concerning their functional relationships with local tissues.
Fat-resident Tregs in obesity-associated metabolic disorders Overwhelming evidence has supported the classification of obesity as a chronic, low-grade inflammatory disease that can promote immunometabolic dysregulation, systemic insulin resistance (IR) and type 2 diabetes (T2D). ${ }^{42}$ Tremendous efforts have been made to uncover the molecular and cellular mechanisms for obesity-induced chronic inflammation, and special attention has been paid to various innate and adaptive immune cells. ${ }^{42}$ Despite the controversies, visceral adipose tissue (VAT) CD4+Foxp3+ regulatory $\mathrm{T}$ cells have been regarded as a key regulator in obesity-associated metabolic disorders. ${ }^{43}$ These cells are abundant in the visceral adipose tissue of normal diet mice, but their proportion is greatly reduced in insulin-resistant animal models of obesity. ${ }^{43,44}$ The depletion of Treg cells led to pro-inflammatory VAT profiles and the worsening of metabolic control in obese mice. ${ }^{43,45}$ To date, several reports have revealed unique properties of VAT Treg cells, including their specific gene expression signatures, transcription factors, distinct chemokine receptors, $\mathrm{T}$ cell receptor (TCR) repertoire and unique functions in adipose tissue.

\section{Characteristics of VAT Tregs}

Compared to conventional Tregs, VAT Tregs behave as a unique subset with distinct characteristics (Table 1).

1. Compared to their counterparts in spleen and lymph nodes, VAT Tregs account for a high ratio (40-60\%) among CD4 $+\mathrm{T}$ cells. Both VAT and subcutaneous fat possess comparable low levels of Tregs at birth, but only the former demonstrates a constant accumulation of Tregs during development. ${ }^{34}$ Strikingly, more than 50\% of the CD4+ cells in the VAT expressed Foxp3, which represents a much higher percentage than that found in the spleen, lymph nodes (LN) (5-20\%) or non-lymphoid tissues (e.g., lung and liver), as well as that found in subcutaneous fat. ${ }^{34}$

2. IL-10 was highly elevated at the transcriptional level in VAT Tregs. The transcriptional level of IL-10 was increased almost 136-fold in VAT Tregs compared to those of the LN, spleen and lung. Further analysis suggested that VAT Tregs produce high levels of IL-10 and exhibit upregulation of genes downstream of the IL-10 receptor. ${ }^{34}$ In turn, the IL-10 produced by VAT Tregs suppresses the inflammatory cytokines and chemokines induced by TNF- $\alpha$, increases GLUT4 expression in adipocytes and restores insulin sensitivity, thus playing vital roles in the prevention of obesityassociated metabolic disorders. ${ }^{34}$

3. VAT Tregs retain approximately $60 \%$ of conventional Tregs markers. Although gene-expression profiling and TCR repertoire analysis revealed that VAT Tregs as a population were distinct from their counterparts in other lymphoid and/or non-lymphoid organs, these cells reserved up to $60 \%$ of the intact signatures of conventional Tregs, including CD25, Foxp3, GITR, 
Table 1 Key properties of Tregs in non-lymphoid and lymphoid tissues

\begin{tabular}{|c|c|c|c|}
\hline & VAT Tregs & Muscle Tregs & lymphoid tissues \\
\hline Frequencies(among the CD4+ T cells) & $40-60 \%$ & About $50 \%$ & $5-20 \%$ \\
\hline Cytokine production & IL-10, TGF- $\beta$ & $\begin{array}{l}\text { IL-10, PGDF, AREG, Granzyme } \\
\text { B }\end{array}$ & $\begin{array}{l}\text { IL-10, TGF- } \beta \text {, IL-35, Granzyme A/B, } \\
\text { perforin }\end{array}$ \\
\hline Phenotype & $\begin{array}{l}\text { With } 60 \% \text { signatures of conventional } \\
\text { Tregs, including CD25, Foxp3, GITR, } \\
\text { CTLA-4, OX40, CD36 and KIrg1 }\end{array}$ & 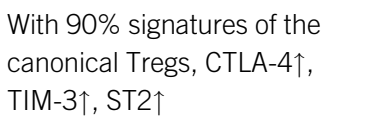 & $\begin{array}{l}\text { CD25, CD127, CD39, CD73, GITR, } \\
\text { CTLA-4, LAG3, ICOS, Neuropilin-1 }\end{array}$ \\
\hline T cell receptor(TCR) repertoire & Specific TCR repertoire & Repeated TCR repertoire & Diverse \\
\hline Specific transcriptional factor & Foxp3, PPAR $-\gamma$ & Foxp3, Stab1 $\downarrow$ & Foxp3 \\
\hline Chemokine & $\begin{array}{l}\text { High levels of CCR1, CCR2, CCR9, } \\
\text { CCL6, GM1960 (an IL-10-inducible } \\
\text { CXCR2 ligand), alcam, CXCL2 and } \\
\text { CXCL10; low levels of CCL5 and CXCR3 }\end{array}$ & $\begin{array}{l}\text { Chemokine receptor (e.g. } \\
\text { CCR } 1 \text { ), }\end{array}$ & CCR6 \\
\hline Function & $\begin{array}{l}\text { Anti-inflammation, ameliorate insulin } \\
\text { resistance, }\end{array}$ & $\begin{array}{l}\text { Promote differentiation of } \\
\text { satellite cells; regulate the } \\
\text { switches of myeloid cells; } \\
\text { promote muscle regeneration }\end{array}$ & $\begin{array}{l}\text { Maintain immunological self- } \\
\text { tolerance; anti-inflammation; establish } \\
\text { transplantation tolerance }\end{array}$ \\
\hline
\end{tabular}

Table1 summarizes the main differences inTregs from VAT, muscle and lymphoid tissues. Compared to Tregsin lymphoid tissues, VAT and muscle Tregs display high frequencies of unique cell markers in addition to most of the signature of conventional Tregs, distinct TCRs, specific transcriptional factors, and chemokines; they also perform special functions in local tissues.

CTLA-4, OX40 and killer cell lectin-like receptor G1 $(\operatorname{KIrg} 1){ }^{34}$

4. VAT is enriched in chemokines involved in leukocyte migration and extravasation.Studies showed that a large set of chemokines and chemokine receptors were highly expressed by VAT Tregs, including CCR1, CCR2, CCR9, CCL6, GM1960 (an IL-10-inducible CXCR2 ligand), alcam, CXCL2 and CXCL10. However, VAT Tregs expressed low levels of CCL5 and CXCR $3 .{ }^{33,34}$ Although the mechanism by which Tregs exert their function via these chemokine/chemokine receptors remains elusive, it could be hypothesized that peripheral Tregs and leukocytes (especially anti-inflammatory leukocytes) may be recruited to VAT based on their unique pattern of chemokine-chemokine receptor expression.

5. PPAR- $\gamma$ is essential for modulating VAT Tregs accumulation, phenotype and function. ${ }^{25}$ Nuclear receptor peroxisome proliferator-activated receptor gamma (PPAR- $\gamma$ ), an adipose tissue-specific transcription factor, exerts multiple functions in adipose tissue. Upon activation, PPAR- $\gamma$ in mature adipocytes can normalize insulin sensitivity. VAT Tregs showed high levels of PPAR- $\gamma$, which is closely related to their unique function in adipose tissue. Specific deletion of PPAR- $\gamma$ in Tregs impeded the accumulation of Tregs in adipose tissue but not in the lymphoid organs and decreased almost all of the Treg signatures of VAT Tregs. ${ }^{34}$ Furthermore, insulin sensitivity in obese mice could be restored by upregulation of $P P A R-\gamma$, which can maintain the particular phenotype of VAT Tregs by interacting with Foxp3. ${ }^{25}$

5. PPAR- $\gamma$ ligands can upregulate the expression of CD36 on macrophages, and thus stimulate oxidized low-density lipoprotein uptake. ${ }^{46}$ Various metabolites or nutrients were recently reported to regulate Tregs generation, trafficking, and function. ${ }^{4-50}$ Furthermore, in contrast to effector $\mathrm{T}$ cells, Tregs preferentially use exogenous fatty acids as a substrate for oxidation, and their differentiation selectively relies on lipid metabolism but not glucose. ${ }^{51}$ Thus, PPAR- $\gamma$ in adipose tissues could enhance the expression of lipid scavenger CD36, there by accelerating the uptake of lipids by VAT Tregs. Furthermore, CD36 may be a critical mediator for PPAR- $\gamma$ regulation of the unique functions of VAT Tregs. ${ }^{52}$

6. VAT Tregs express specific $\mathrm{T}$ cell receptor repertoires. The $\mathrm{T}$ cell receptor (TCR) repertoire is regarded as another criterion for the evaluation of the similarity of $\mathrm{T}$ cell populations. The TCR repertoires of VAT Tregs differed from those of LN Tregs and conventional T cells, indicating that VAT Tregs were not likely to be a product of the influx or retention of peripheral Tregs of a particular specificity or the conversion of local cytokine-induced T cells. ${ }^{34}$ Moreover, the presence of repeated VAT Tregs TCR clones suggests that specific antigen(s) may exist and be responsible for VAT Tregs accumulation. ${ }^{33}$ Further analysis of the TCR repertoire will help to clarify the origin, development and localization of VAT Tregs.

\section{Function of VAT Tregs in obesity-associated metabolic disorders and insulin resistance}

VAT-specific antigen(s) recognition and expression of specific chemokine receptors may mediate the recruitment and retention of Treg cells in adipose tissue. To date, the VAT-restricted antigens for VAT Treg cells recognition, accumulation and retention remain unknown. It is possible that $\mathrm{CD} 4+\mathrm{CD} 25+$ Foxp3+ Tregs in VAT draining lymph nodes could be functionally activated by VAT-restricted antigenic ligands of mature DCs and subsequently survive and migrate into fat 


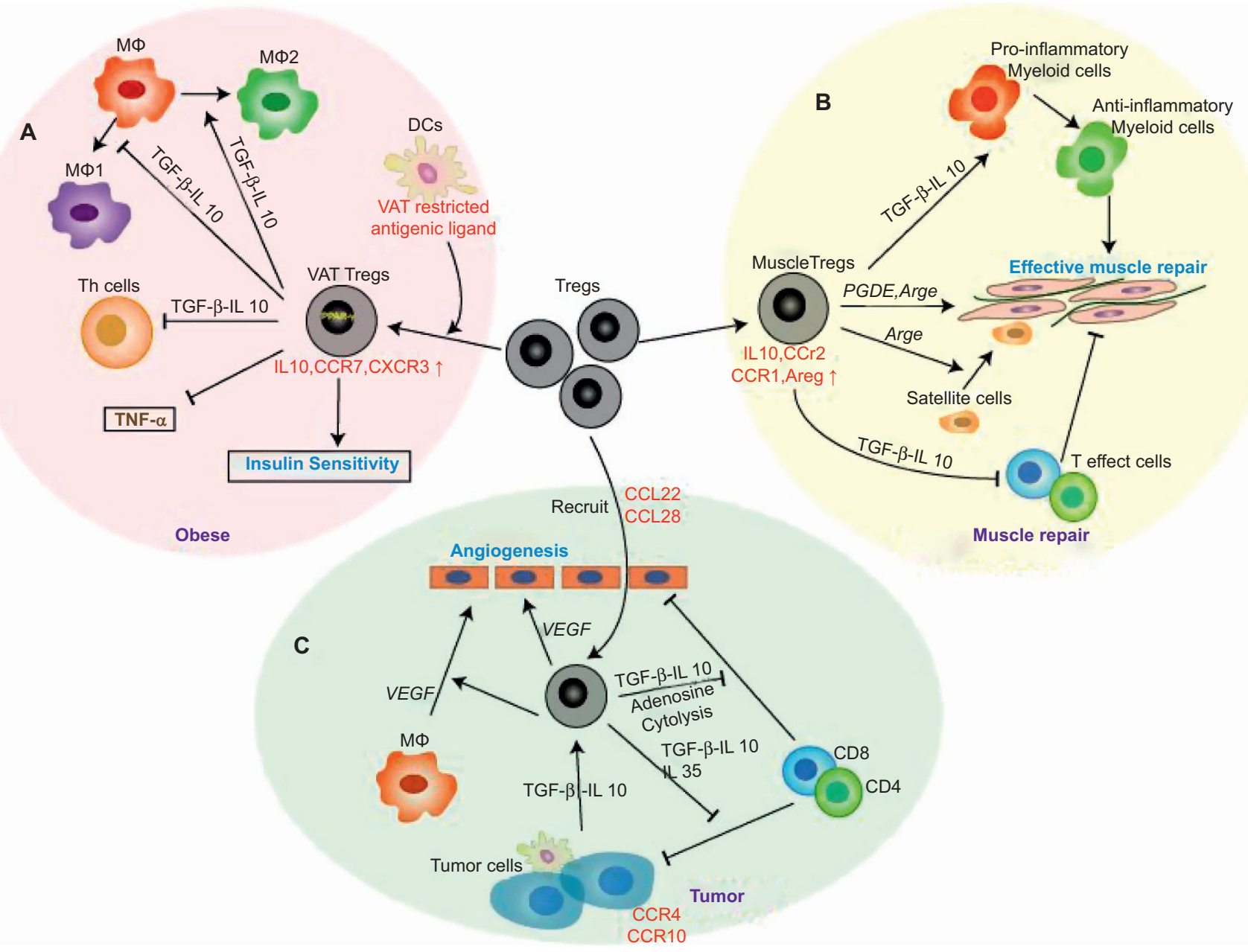

Figure 1 Functions of resident Tregs in obesity-associated metabolic disorders, muscle repair and tumor progress. a. VAT Tregs. Tregs in VAT draining lymph nodes display an anti-inflammatory capacity in obesity-associated metabolic disorders. ${ }^{54,55}$ Functionally, VAT Tregs suppress Th cells (i.e., Th1, Th2, and Th17) by secreting anti-inflammatory IL-10 and TGF- $\beta^{34}$ and preventing the conversion of macrophages to type-1 microphages. ${ }^{25,34}$ Alternatively, VAT Tregs directly attenuate the negative roles of TNF- $\alpha$ in inflammation in adipose tissue ${ }^{33}$ and restore insulin sensitivity in obese mice via up regulating PPAR- $\gamma .{ }^{25,54}$ b. Muscle Tregs. Muscle Tregs potentiate muscle repair through IL-10, CCR1, PGDF and amphiregulin (AREG). ${ }^{26}$ Muscle Tregs pose as a barrier to the transitions of myeloid cells from a pro- to an anti-inflammatory phenotype and accelerate the differentiation of satellite cells, thereby promoting the regeneration of skeletal muscle. ${ }^{26,66}$ Moreover, the PDGF and Areg produced by muscle Tregs also directly contribute to muscle repair. ${ }^{26}$ c. Tumoral Tregs. Tregs can infiltrate into tumor tissues via the chemo-attractive CCR4-CCL22 and CCR10-CCL28 axis.Tregs promote tumor progression by inhibiting antitumor effector T cells via producing inhibitory cytokines and cytolysis. ${ }^{24}$ TGF- $\beta$ and IL-10 produced by tumor cells also convert CD4+ naive precursors into induced Tregs, which aggravate the immune escape. Tregs further contribute to the angiogenesis required for tumor growth by enhancing VEGF production directly or through their effects on macrophages. ${ }^{84-86}$

tissues, where re-stimulations by VAT antigens confer Tregs anti-inflammatory functions in healthy lean mice.

VAT Tregs may efficiently harbor anti-inflammatory functions in IR and metabolic disorders secondary to obesity (Figure 1). Recent studies revealed that high levels of VAT Tregs in female mice protected the mice from high fat diet (HFD)-induced metabolic disorders compared with male mice. ${ }^{53}$ Moreover, the deletion of VAT Tregs caused a severe loss ininsulin sensitivity and upregulated the levels of inflammatory mediators in the visceral fat. ${ }^{33}$ Consistently, the induction of CD4+LAP+Tregs in vivo by oral administration of CD3 and $\beta$-glucosylceramide, ${ }^{54}$ the expansion of VAT Tregs by the IL-2 /anti-IL-2 complex, ${ }^{33}$ or the adoptive transfer of CD4 + CD25+ Tregs significantly improved insulin sensitivity in obese mice. ${ }^{45,54,55}$

Moreover, the expansion of VAT Tregs in mice with HFDinduced metabolic disorders by the IL-2/anti-IL-2 complex contributed to the reduction in weight gain, adipocyte size and epididymal fat. ${ }^{34,56}$ Increased adiposity and decreased insulin sensitivity were found to be correlated with decreased populations of VAT Tregs. Other studies have shown that high-levels of VAT Tregs were associated with alleviated inflammation and the recovery of IR without affecting weight or the numbers and sizes of adipocytes. ${ }^{44,54}$ 
Therefore, questions have arisen concerning whether VAT Tregs affect the hyperplasia and hypertrophy of adipocytes. Insightful explorations into the mechanisms of VAT Tregs activity are vital for the manipulation of resident Tregs in the clinical therapy of $\mathrm{T} 2$.

\section{A UNIQUE POPULATION OF TREGS IN SKELETAL MUSCLE}

Recently, a unique Tregs population that contributes to muscle repair was identified in injured skeletal muscle. ${ }^{26}$ This population displays a special TCR repertoire, exhibits a distinct transcriptome and exerts unique functions. In a dystrophic animal model, punctual depletion of Tregs during the repair process increased muscle damage; similarly, enhancement of Tregs function decreased the pathology, ${ }^{26}$ indicating a crucial role for local Tregs in muscle damage and repair.

\section{Characteristics of muscle Tregs}

Despite some critical characteristics similar to classical lymphoid Tregs, muscle Tregs possess some unique features that are distinct from Tregs in lymphoid organs (Table 1).

Once skeletal muscle was injured, Tregs began to infiltrate and accumulate locally within days.The frequency of muscle Tregs among CD4 $+\mathrm{T}$ cells increased steadily to $\sim 50 \%$, which is much higher than the $5-20 \%$ frequency of lymphoid Tregs; the frequency remained at that level for more than 30 days. ${ }^{26}$

In addition to possessing $91 \%$ of the canonical Tregs signature, ${ }^{26}$ the gene expression profile of muscle Tregs indicates that they also possess unique traits. A set of genes are unique to muscle Tregs, including anti-inflammatory cytokine IL-10, chemokine receptors (e.g., CCR1), PGDF and amphiregulin (AREG); these genes contribute to the function of muscle Tregs in the context of regenerating muscle. ${ }^{26}$ Other elements that distinguish muscle Tregs from lymphoid-organ Tregs include decreased expression of Satb1 and enhanced levels of ST2, Gzmb, CTLA-4, and TIM-3. ${ }^{2620}$ Satb1 was reported to encode a chromatin organizer, and the inhibition of Satb1 is crucial for the Tregs phenotype and suppressive function. ${ }^{57}$ Tregs in dystrophic muscle also express high levels of ST2, which was reported to mediate $\mathrm{T}$ cell polarization. ST2 + Tregs were recently shown to represent an activated subset of Foxp $3+$ cells; $^{58}$ this finding is consistent with the data that muscle Tregs in necrotic lesions displayed an activated phenotype. ${ }^{59}$ Thus, ST2+ Treg cells in damaged muscles may possess unique biological functions and facilitate muscle repair in addition to their immune suppressive functions.

Muscle Tregs also display a unique repertoire of TCRs. This unique TCR repertoire that has never been detected in spleen Tregs and conventional muscle $\mathrm{T}$ cells has been reported for muscle Tregs. Recently, it was reported that AREG could markedly enhance Tregs activity and was critical for the efficient suppressive function of Tregs. ${ }^{26}$ Interestingly, it was demonstrated that a small subset of Areg+ Tregs in the spleen shared TCR sequences with both Areg-positive and -negative Tregs from muscle. These findings indicate that Tregs in injured muscle may be clonally expanded and that some muscle antigens might be involved in recruiting Tregs to the injured site and/or retaining them therein. ${ }^{26}$

\section{Local Tregs Help Muscle Repair}

Once damaged, skeletal muscle regeneration follows the same orchestrated program as other organ regeneration. Muscle injury initiates a series of responses by specific myeloid cell populations; this process is initially dominated by neutrophils and subsequently by CD68+ M1 macrophages. The phenotypic and functional transitions of these myeloid cells from a pro- to an anti-inflammatory phenotype are crucial for proper muscle repair. ${ }^{60}$ Tregs contribute to tissue repair by promoting the polarization of anti-inflammatory M2 macrophages. ${ }^{61,62}$ Recent evidence also showed that Tregs in dystrophic muscles increased the expression of IL-10, an important mediator thatcan induce the differentiation of M2 macrophages. ${ }^{59,62}$ Furthermore, the depletion of Tregs exacerbated muscle injury due to anenhanced IFN- $\gamma$ responses and the activation of M1 macrophages, while the expansion of Tregs with IL-2/anti-IL-2 complexes increased the levels of IL-10 in muscle, resulting in decreased myofiber injury. ${ }^{59}$ Therefore, Tregs may regulate the myeloid populations that infiltrate damaged tissues and promote their beneficial switches. ${ }^{26}$ It is also possible that the loss of Tregs impedes the muscle repair process, at least in part, by blocking the anti-inflammatory M2 polarization.

Skeletal muscle regeneration is largely driven by satellite cells that represent an endogenous source of muscle progenitor cells with the regenerative potential of adult muscle. ${ }^{63}$ It has been reported that muscle Tregs can promote the differentiation of satellite cells in vitro and in vivo via the overexpression of the EGF family member Areg, ${ }^{26}$ which promotes the regeneration of multiple tissues, including the intestinal mucosa and lung epithelium. ${ }^{64,65}$ Furthermore, previous studies revealed that Areg might act directly on muscle cells. ${ }^{66}$

Aside from their activities on muscle-lineage cells, muscle Tregs also regulate conventional T cells. Elevated frequencies of muscle Tregs at the injury site significantly decreased Tconv (and/or CD8 + and B cells) in a mouse model, thereby contributing to muscle repair. ${ }^{26}$

Collectively, muscle Tregs indirectly and directly contribute to tissue regeneration by dual mechanisms (Figure 1).These Tregs regulate the infiltration and transitions of myeloid cells and produce Areg to promote the differentiation of satellite cells; however, they also suppress the function of Tconv cells and exert their function to improve muscle repair. Thus, this population of Tregs may provide new therapeutic opportunities for muscle repair.

\section{TUMORAL TREGS AND ANGIOGENESIS}

Tumors escape from immune surveillance by establishing potent, overlapping mechanism. ${ }^{67-70}$ Tregs are highly enriched in a variety of tumor types that are often associated with a poor prognosis. ${ }^{71}$ Tumor-infiltrating Tregs are considered to be potent immunosuppressive factors that promote the progression of cancers, ${ }^{71}$ while intra-tumoral $\mathrm{T}$ cells have been demonstrated to play antitumor roles. ${ }^{72}$ Soluble suppressive cytokines 
produced by Tregs, including IL-10, TGF- $\beta$ and IL-35, inhibit the anti-tumor functions of effectors, thereby promoting tumor progression. Of note, recent findings demonstrated that tumor Tregs produce VEGF to promote angiogenesis and directly contribute to tumor growth, ${ }^{24}$ expanding the functional spectrum of tumoral Tregs.

\section{Tumor angiogenesis and immune escape}

Blood vessel development is critical to tumor growth because cancer cells rely on angiogenesis to fulfill their need for oxygen and growth factors. ${ }^{73}$ Moreover, tumor angiogenesis impairs the interactions between immune cells and the vessel wall, contributing to an immunosuppressive tumor microenvironment that enables tumors to evade host immunosurveillance. ${ }^{74,75}$ Strategies that reduce angiogenesis increased the numbers of tumor-infiltrating $\mathrm{T}$ cells and improved immune therapeutic results. ${ }^{76-80}$ These explorations provide attractive rationales for the potential to combine anti-angiogenic agents with immune treatment for cancer patients.

VEGF is produced by many tumors and has been shown to play key roles in tumor angiogenesis, growth and metastasis. ${ }^{81}$ Furthermore, VEGF has been shown to contribute to the induction of Tregs in patients with cancer. ${ }^{82}$ The presence of Foxp3+ lymphocytes was significantly associated with high intra-tumoral microvessel density and high levels of VEGF and other markers for angiogenesis in endometrial and breast tumors, ${ }^{83,84}$ providing further evidence that Tregs may contribute to tumor angiogenesis. Tregs depletion enhanced anti-tumor CD4 responses by increasing IFN- $\gamma$ production, which exerts a potent anti-angiogenesis function. ${ }^{85} \mathrm{~A}$ recent study further showed that tumor hypoxia induced CCL28 expression, leading to the recruitment of CCR10+ Tregs. ${ }^{24}$ CD4+CD25+ Tregs secreted higher amounts of VEGF undernormal oxygen conditions and hypoxic conditions compared with CD4+CD25-T cells. The recruitment of Tregs established a VEGFA-rich tumor microenvironment and increased tumor angiogenesis, whereas the depletion of Tregs with anti-CD25 antibodies or the blockade of CCR10 reduced tumor VEGFA levels and tumor vascularization. ${ }^{24}$ The positive correlation between the presence of Foxp3+ Tregs and immature tumor angiogenesis was also confirmed in renal cell carcinomas. ${ }^{86}$ These studies increased our understanding of the characteristics of Tregs and highlighted a multifaceted role for Tregs in promoting cancer growth through both tumor immune escape and angiogenesis (Figure 1).

\section{Tregs as targets for cancer immunotherapy}

Many strategies have been used to manipulate the behavior of Tregs in a variety of diseases, including Tregs depletion, inhibition of tregs function, and the blockade of Tregs trafficking, signaling and anti-angiogenic pathways. ${ }^{30}$ Recently, new signaling targets to block Tregs and/or boost $\mathrm{T}$ effectors are being evaluated in clinical trials.

With the novel findings of new roles for tumor Tregs, new targets to manipulate Tregs should be explored for designing future therapies to treat tumors. ${ }^{87}$ The positive correlation between the presence of Foxp3 + Tregs and tumor angiogenesis has been demonstrated for many types of tumors. ${ }^{88,89}$ Tregs in hypoxic tumor microenvironments secreted large amounts of VEGF, and depletion of Tregs or blockade of Tregs migration reduced tumor VEGFA levels and tumor vascularization. ${ }^{24}$ Given the critical role of Tregs in tumor immune suppression and the promotion of angiogenesis, targeting Tregs is one vital approach for clinical tumor treatment.

Accumulating evidence strongly suggests that antiangiogenesis treatments overcome various immunosuppressive networks. Sunitinib, an inhibitor of the tyrosine kinase involved in angiogenesis, represents a front line therapy for the treatment of metastatic renal cell carcinoma (mRCC) and has been reported to reduce the percentage and absolute number of Tregs. ${ }^{90}$ Moreover, the measurement of Tregs may shed light on the prognosis of the response of the patient to antiangiogenic therapy ${ }^{89,90}$ Therefore, antiangiogensis therapies exert their function through both inhibiting vascularization and regulating the immune responses. In turn, strategies to block Tregs may also affect the progress of angiogenesis. The combination of multiple strategies, such as anti-angiogenesis therapy with immune approaches, may provide a promising prospect for the treatment of cancer patients in the near future.

\section{TREG CELLS IN BONE HOMEOSTASIS}

The human skeleton is subjected to continuous remodeling during its lifespan. An imbalance in osteoblast-mediated bone formation and osteoclast-mediated bone resorption leads to the degradation of bone. Immune activation can trigger this imbalance. Several studies have found that Tregs can directly control bone resorption in vivo and preserve bone mass during physiological and pathological bone remodeling. ${ }^{32,91}$ Previously, studies demonstrated that the addition of Tregs to monocyte cultures inhibited the differentiation of monocytes into osteoclasts in vitro. ${ }^{92,93}$ Foxp3-Tg mice showed impaired osteoclast differentiation and bone resorption, developed higher bone mass and were protected from ovariectomy-induced bone loss in vivo. ${ }^{91}$ Furthermore, adoptive transfer of CD4+CD25+ T cells into T cell-deficient RAG-1-/-mice also increased bone mas. ${ }^{91}$ Consistently, the expansion of Tregs in vivo by a CD28 super antibody suppressed local and systemic bone destruction and increased bone density in healthy mice. These studies indicated that Treg cells directly affect bone homeostasis and that this function was independent of their suppression of inflammation.

The mechanisms by which Tregs exert their functions on bone have not been fully illustrated. Cell-cell contact via CTLA-4 has been shown to be essential for the blockade of osteoclast formation by Tregs. ${ }^{36}$ A recent study showed that injection of CD4+ iTregs markedly suppressed osteoclastogenesis ina collagen-induced arthritis (CIA) mouse modelin a cellcontact but not cytokine-dependent manner. ${ }^{94}$ Although bone-specific Tregs have not been proven to exist to date, the accumulation of Tregs at sites of intense bone remodeling (i.e., the epiphysis of long bones) favors the concept that Treg cells need cell-to-cell contact and act locally on bone. Further studies are needed to clarify the existence and function 
of bone-resident Tregs, and thus extend our knowledge and drive the development of immune tools to maintain bone mass.

\section{SKIN MEMORY TREGS IN HUMANS}

The skin acts as a well-characterized barrier between the body and the environment and provides the first line of immunological defense against infections and injuries. Extensive crosstalk between the epithelial, stromal and immune cells plays vital roles in maintaining immune homeostasis in skin. ${ }^{95}$ Of note, recent studies demonstrated that skin-resident memory $\mathrm{T}$ cells achieved superior protection compared with circulating memory $\mathrm{CD} 4+\mathrm{T}$ cells, indicating that the response to specific antigens effect the functions of T cells in tissues. ${ }^{96}$ In addition to skin memory $\mathrm{T}$ cells, skin resident memory Treg cells (mTregs) in mice and humans have recently been identified. ${ }^{97-99}$ These activated Treg cells persisted in the skin and suppressed autoimmune responses upon repeated exposure to self-antigen. ${ }^{97}$ These discoveries provided mechanistic insight into the crosstalk between tissues and immunity.

Similar to other tissue Tregs subsets in mice, human skinresident Tregs possess their own characteristics, including phenotype, function and localization. mTreg in skin express high levels of activation markers including CTLA-4, CD25 and ICOS compared to CD45RO+CD4+Foxp3+ cells in adult peripheral blood. ${ }^{99}$ TCR $\beta$ sequencing of mTregs and memory $\mathrm{T}$ conventional cells (Tconvs) in human skin showed that these two populations share very few overlapping sequences, suggesting that they predominantly recognize different antigens.

Moreover, both human and murine memory Tregs preferentially localized to hair follicles(HFs). ${ }^{99,100}$ Human mTregs were more abundant in skin with high hair density, ${ }^{99}$ and this phenomenon may correlate with autoimmune diseases such as alopecia areata (AA) that affect HFs. ${ }^{101}$ mTregs have a distinct cytokine profile compared with $\mathrm{T}$ convs. Skin mTregs produce less IL-2 and IL-17 compared to activated Tregs in the blood. Moreover, mTregs proliferate and produce more IL-17 in psoriatic skin compared to Tregs in non-lesional skin. ${ }^{99}$ These results indicate that a deficiency in skin Tregs may closely correlate with autoimmunity and inflammatory skin disease.

To date, new discoveries have provided essential steps towards understanding the nature of Tregs in humans. However, questions concerning the presence of other tissue-specific Tregs and how human resident Tregs exert their function locally still exist. Future studies elucidating the fundamental biology of Tregs in human tissues will be indispensable for defining their abnormalities in diseases and the potential exploitation of these cells to treat diseases.

\section{CONCLUSIONS}

Given the indispensable roles of regulatory $\mathrm{T}$ cells in immune balance and tolerance, a great deal of effort has been focused on elucidating Tregs identity, heterogeneity, stability and function. More recent and emerging evidence has confirmed the existence, characteristics, and functions of tissue-specific Tregs and provided promising perspectives for future therapy.
However, many issues concerning tissue-specific Tregs need to be addressed prior to their use as a drug target for the development of effective therapies.

For example, the possibility of specialized subsets of Tregs in each tissue needs to be addressed. If this is the case, what are the phenotypes and characteristics of these subsets, and how do they exert their functions locally? How can the functions of tissue resident Tregs be distinguished from those that migrate to the tissue? How do these diverse populations orchestrate local homeostasis? What is the mechanism by which tissue resident Tregs are regulated by the host, and how can these populations be manipulated to treat disease? Unraveling the complexity of local Tregs is just the beginning, and further explorations into their characteristics, functions and targets will undoubtedly enhance future therapeutic development.

Table 1. Key properties of Tregs in non-lymphoid and lymphoid tissues.

\section{ACKNOWLEDGMENTS}

This work was supported by the National Natural Science Foundation of China (81273263, 81202332, 81370434,and 81470393), 973 Program (2012CB526605), Shanghai Municipal Program on Key Basic Research Project (11JC1410800,801), Shanghai Science and Technology Development Funds (12QA1402900), SRFDP(20130072120062), Key Disciplines Group Construction Project (PWZxq2014-01) and Outstanding Leaders Training Program of Pudong Health Bureau of Shanghai (PWRl2011-01).

1 Abbas, A.K., C. Benoist, J.A. Bluestone, et al. Regulatory T cells: recommendations to simplify the nomenclature. Nat Immunol 2013; 14: 307-8.

2 Zhou, X., S. Bailey-Bucktrout, L.T. Jeker, and J.A. Bluestone. Plasticity of CD4(+) FoxP3(+) T cells. Curr Opin Immunol 2009; 21: 281-5.

3 Sakaguchi, S., N. Sakaguchi, M. Asano, M. Itoh, and M. Toda. Immunologic self-tolerance maintained by activated $\mathrm{T}$ cells expressing IL-2 receptor alpha-chains (CD25). Breakdown of a single mechanism of self-tolerance causes various autoimmune diseases. J Immunol 1995; 155: 1151-64.

4 Baecher-Allan, C., J.A. Brown, G.J. Freeman, and D.A. Hafler. CD4+CD25high regulatory cells in human peripheral blood. $\mathrm{J}$ Immunol 2001; 167: 1245-53.

5 Ng, W.F., P.J. Duggan, F. Ponchel, et al. Human CD4(+)CD25(+) cells: a naturally occurring population of regulatory T cells. Blood 2001; 98: 2736-44.

6 Jonuleit, H., E. Schmitt, M. Stassen, A. Tuettenberg, J. Knop, and A.H. Enk. Identification and functional characterization of human CD4(+)CD25(+) T cells with regulatory properties isolated from peripheral blood. J Exp Med 2001; 193: 1285-94.

7 Dieckmann, D., H. Plottner, S. Berchtold, T. Berger, and G. Schuler. Ex vivo isolation and characterization of CD4(+)CD25(+) T cells with regulatory properties from human blood. J Exp Med 2001; 193: 1303-10.

8 Hori, S., T. Nomura, and S. Sakaguchi. Control of regulatory T cell development by the transcription factor Foxp3. Science 2003; 299: 1057-61.

9 Walker, M.R., D.J. Kasprowicz, V.H. Gersuk, et al. Induction of FoxP3 and acquisition of T regulatory activity by stimulated human CD4+CD25- T cells. J Clin Invest 2003; 112: 1437-43. 
10 Sakaguchi, S., M. Miyara, C.M. Costantino, and D.A. Hafler. FOXP3+ regulatory T cells in the human immune system. Nat Rev Immunol 2010; 10: 490-500.

11 Liu, W., A.L. Putnam, Z. Xu-Yu, et al. CD127 expression inversely correlates with FoxP3 and suppressive function of human CD4+ T reg cells. J Exp Med 2006; 203: 1701-11.

12 de Vries, I.J, C. Castelli, C. Huygens, et al. Frequency of circulating Tregs with demethylated FOXP3 intron 1 in melanoma patients receiving tumor vaccines and potentially Treg-depleting agents. Clin Cancer Res 2011; 17: 841-8.

13 Medzhitov, R., E.M. Shevach, G. Trinchieri, et al. Highlights of 10 years of immunology in Nature Reviews Immunology. Nat Rev Immunol 2011; 11: 693-702.

14 Korn, T., E. Bettelli, M. Oukka, and V.K. Kuchroo. IL-17 and Th17 Cells. Annu Rev Immunol 2009; 27: 485-517.

15 Haas, J., B. Fritzsching, P. Trubswetter, et al. Prevalence of newly generated naive regulatory $T$ cells (Treg) is critical for Treg suppressive function and determines Treg dysfunction in multiple sclerosis. J Immunol 2007; 179: 1322-30.

16 Horwitz, D.A., S.G. Zheng, J. Wang, and J.D. Gray. Critical role of IL2 and TGF-beta in generation, function and stabilization of Foxp3+CD4+ Treg. Eur J Immunol 2008; 38: 912-5.

17 Xie, J.J., J. Wang, T.T. Tang, et al. The Th17/Treg functional imbalance during atherogenesis in $\mathrm{Apo} \mathrm{E}(-/-)$ mice. Cytokine 2010; 49: 185-93.

18 Yang, X.O., R. Nurieva, G.J. Martinez, et al. Molecular antagonism and plasticity of regulatory and inflammatory $\mathrm{T}$ cell programs. Immunity 2008; 29: 44-56.

$19 \mathrm{Xu}$, L., A. Kitani, I. Fuss, and W. Strober. Cutting edge: regulatory $T$ cells induce $C D 4+C D 25-$ Foxp $3-T$ cells or are selfinduced to become Th17 cells in the absence of exogenous TGFbeta. J Immunol 2007; 178: 6725-9.

20 Zhou, X., N. Kong, J. Wang, et al. Cutting edge: all-trans retinoic acid sustains the stability and function of natural regulatory $T$ cells in an inflammatory milieu. J Immunol 2010; 185: 2675-9.

21 Lu, L., Q. Lan, Z. Li, et al. Critical role of all-trans retinoic acid in stabilizing human natural regulatory $T$ cells under inflammatory conditions. Proc Natl Acad Sci U S A 2010; 111: E3432-40.

22 Vignali, D.A., L.W. Collison, and C.J. Workman. How regulatory T cells work. Nat Rev Immunol 2008; 8: 523-32.

23 Deaglio, S., K.M. Dwyer, W. Gao, et al. Adenosine generation catalyzed by CD39 and CD73 expressed on regulatory T cells mediates immune suppression. J Exp Med 2007; 204: 1257-65

24 Facciabene, A., X. Peng, I.S. Hagemann, et al. Tumour hypoxia promotes tolerance and angiogenesis via CCL28 and T(reg) cells. Nature 2011; 475: 226-30.

25 Cipolletta, D., M. Feuerer, A. Li, et al. PPAR-gamma is a major driver of the accumulation and phenotype of adipose tissue Treg cells. Nature 2012; 486: 549-53.

26 Burzyn, D., W. Kuswanto, D. Kolodin, et al. A special population of regulatory T cells potentiates muscle repair. Cell 2013; 155 : 1282-95.

27 Huber, S., N. Gagliani, E. Esplugues, et al. Th17 cells express interleukin-10 receptor and are controlled by Foxp3(-) and Foxp3+ regulatory CD4+ T cells in an interleukin-10-dependent manner. Immunity 2011; 34: 554-65.

28 Cao, X., S.F. Cai, T.A. Fehniger, et al. Granzyme B and perforin are important for regulatory $\mathrm{T}$ cell-mediated suppression of tumor clearance. Immunity 2007; 27: 635-46.

29 Pandiyan, P., L. Zheng, S. Ishihara, J. Reed, and M.J. Lenardo. CD4 + CD25+Foxp3 + regulatory T cells induce cytokine deprivation-mediated apoptosis of effector CD4+ T cells. Nat Immunol 2007; 8: 1353-62.

30 Pere, H., C. Tanchot, J. Bayry, et al. Comprehensive analysis of current approaches to inhibit regulatory $T$ cells in cancer. Oncoimmunology 2012; 1: 326-333.

31 Wing, K., Y. Onishi, P. Prieto-Martin, et al. CTLA-4 control over Foxp3+ regulatory T cell function. Science 2008; 322: 271-5.
32 Zaiss, M.M., B. Frey, A. Hess, et al. Regulatory T cells protect from local and systemic bone destruction in arthritis. J Immunol 2010; 184: 7238-46.

33 Cipolletta, D., D. Kolodin, C. Benoist, and D. Mathis. Tissular $T$ (regs): a unique population of adipose-tissue-resident Foxp3+CD4+ T cells that impacts organismal metabolism. Semin Immunol 2011; 23: 431-7.

34 Feuerer, M., L. Herrero, D. Cipolletta, et al. Lean, but not obese, fat is enriched for a unique population of regulatory $T$ cells that affect metabolic parameters. Nat Med 2009; 15: 930-9.

35 Chen, Z., A.E. Herman, M. Matos, D. Mathis, and C. Benoist. Where CD4+CD25+ T reg cells impinge on autoimmune diabetes. J Exp Med 2005; 202: 1387-97.

36 Nguyen, L.T., J. Jacobs, D. Mathis, and C. Benoist. Where FoxP3dependent regulatory $T$ cells impinge on the development of inflammatory arthritis. Arthritis Rheum 2007; 56: 509-20.

37 Sather, B.D., P. Treuting, N. Perdue, et al. Altering the distribution of Foxp3(+) regulatory $T$ cells results in tissue-specific inflammatory disease. J Exp Med 2007; 204: 1335-47.

38 Tilburgs, T., D.L. Roelen, B.J. van der Mast, et al. Evidence for a selective migration of fetus-specific CD4+CD25bright regulatory $\mathrm{T}$ cells from the peripheral blood to the decidua in human pregnancy. J Immunol 2008; 180: 5737-45.

39 Feuerer, M., Y. Shen, D.R. Littman, C. Benoist, and D. Mathis. How punctual ablation of regulatory $T$ cells unleashes an autoimmune lesion within the pancreatic islets. Immunity 2009; 31: 654-64.

40 Tanchot, C., M. Terme, H. Pere, et al. Tumor-infiltrating regulatory $\mathrm{T}$ cells: phenotype, role, mechanism of expansion in situ and clinical significance. Cancer Microenviron 2013; 6 : 147-57.

41 Rosenblum, M.D., I.K. Gratz, J.S. Paw, K. Lee, A. MarshakRothstein, and A.K. Abbas. Response to self antigen imprints regulatory memory in tissues. Nature 2012; 480: 538-42.

42 Lumeng, C.N. and A.R. Saltiel. Inflammatory links between obesity and metabolic disease. J Clin Invest 2011; 121: 2111-7.

43 Chen, X., Y. Wu, and L. Wang. Fat-resident Tregs: an emerging guard protecting from obesity-associated metabolic disorders. Obes Rev 2013; 14: 568-78.

44 Winer, S., Y. Chan, G. Paltser, et al. Normalization of obesityassociated insulin resistance through immunotherapy. Nat Med 2009; 15: 921-9.

45 Eller, K., A. Kirsch, A.M. Wolf, et al. Potential role of regulatory $T$ cells in reversing obesity-linked insulin resistance and diabetic nephropathy. Diabetes 2011; 60: 2954-62.

46 Tontonoz, P., L. Nagy, J.G. Alvarez, V.A. Thomazy, and R.M. Evans. PPARgamma promotes monocyte/macrophage differentiation and uptake of oxidized LDL. Cell 1998; 93: 241-52.

47 Zeng, $\mathrm{H}$. and $\mathrm{H}$. Chi. Metabolic control of regulatory $\mathrm{T}$ cell development and function. Trends Immunol 2015; 36: 3-12.

48 Furusawa, Y., Y. Obata, S. Fukuda, et al. Commensal microbederived butyrate induces the differentiation of colonic regulatory T cells. Nature 2013; 504: 446-50.

49 Arpaia, N., C. Campbell, X. Fan, et al. Metabolites produced by commensal bacteria promote peripheral regulatory $\mathrm{T}$-cell generation. Nature 2013; 504: 451-5.

50 Smith, P.M., M.R. Howitt, N. Panikov, et al. The microbial metabolites, short-chain fatty acids, regulate colonic Treg cell homeostasis. Science 2013; 341: 569-73.

51 Michalek, R.D., V.A. Gerriets, S.R. Jacobs, et al. Cutting edge: distinct glycolytic and lipid oxidative metabolic programs are essential for effector and regulatory CD4+ T cell subsets. $\mathrm{J}$ Immunol 2011; 186: 3299-303.

52 Sugii, S., P. Olson, D.D. Sears, et al. PPARgamma activation in adipocytes is sufficient for systemic insulin sensitization. Proc Natl Acad Sci U S A 2009; 106: 22504-9.

53 Pettersson, U.S, T.B. Walden, P.O. Carlsson, L. Jansson, and M. Phillipson. Female mice are protected against high-fat diet induced metabolic syndrome and increase the regulatory $\mathrm{T}$ cell population in adipose tissue. PLoS One 2012; 7: e46057. 
54 Ilan, Y., R. Maron, A.M. Tukpah, et al. Induction of regulatory T cells decreases adipose inflammation and alleviates insulin resistance in ob/ob mice. Proc Natl Acad Sci U S A 2010; 107: 9765-70.

55 Osborn, O. and J.M. Olefsky. The cellular and signaling networks linking the immune system and metabolism in disease. Nat Med 2012; 18: 363-74.

56 Svec, P., B. Vasarhelyi, B. Paszthy, et al. Do regulatory T cells contribute to Th1 skewness in obesity? Exp Clin Endocrinol Diabetes 2007; 115: 439-43.

57 Beyer, M., Y. Thabet, R.U. Muller, et al. Repression of the genome organizer SATB1 in regulatory T cells is required for suppressive function and inhibition of effector differentiation. Nat Immunol 2011; 12: 898-907.

58 Bending, D., A.M. Pesenacker, S. Ursu, et al. Hypomethylation at the regulatory T cell-specific demethylated region in CD25hi T cells is decoupled from FOXP3 expression at the inflamed site in childhood arthritis. J Immunol 2014; 193: 2699-708.

59 Villalta, S.A., W. Rosenthal, L. Martinez, et al. Regulatory T cells suppress muscle inflammation and injury in muscular dystrophy. Sci Transl Med 2014; 6: 258ra142.

60 Tidball, J.G. and S.A. Villalta. Regulatory interactions between muscle and the immune system during muscle regeneration. Am J Physiol Regul Integr Comp Physiol 2010; 298: R1173-87.

61 Tiemessen, M.M., A.L. Jagger, H.G. Evans, M.J. van Herwijnen, S. John, and L.S. Taams. CD4+CD25+Foxp3+ regulatory T cells induce alternative activation of human monocytes/macrophages. Proc Natl Acad Sci U S A 2007; 104: 19446-51.

62 Weirather, J., U.D. Hofmann, N. Beyersdorf, et al. Foxp3+ CD4+ T cells improve healing after myocardial infarction by modulating monocyte/macrophage differentiation. Circ Res 2014; 115: 55-67.

63 Goldring, K., T. Partridge, and D. Watt. Muscle stem cells. J Pathol 2002; 197: 457-67.

64 Shao, J. and H. Sheng. Amphiregulin promotes intestinal epithelial regeneration: roles of intestinal subepithelial myofibroblasts. Endocrinology 2010; 151: 3728-37.

65 Monticelli, L.A., G.F. Sonnenberg, M.C. Abt, et al. Innate lymphoid cells promote lung-tissue homeostasis after infection with influenza virus. Nat Immunol 2011; 12: 1045-54.

66 Golding, J.P., E. Calderbank, T.A. Partridge, and J.R. Beauchamp. Skeletal muscle stem cells express anti-apoptotic ErbB receptors during activation from quiescence. Exp Cell Res 2007; 313 : 341-56.

67 Zou, W. Immunosuppressive networks in the tumour environment and their therapeutic relevance. Nat Rev Cancer 2005; 5: 263-74.

68 Curiel, T.J., G. Coukos, L. Zou, et al. Specific recruitment of regulatory $T$ cells in ovarian carcinoma fosters immune privilege and predicts reduced survival. Nat Med 2004; 10: 942-9.

69 Nagaraj, S., K. Gupta, V. Pisarev, et al. Altered recognition of antigen is a mechanism of CD8+ T cell tolerance in cancer. Nat Med 2007; 13: 828-35.

70 Dolcetti, L., I. Marigo, B. Mantelli, E. Peranzoni, P. Zanovello, and V. Bronte. Myeloid-derived suppressor cell role in tumor-related inflammation. Cancer Lett 2008; 267: 216-25.

71 Facciabene, A., G.T. Motz, and G. Coukos. T-regulatory cells: key players in tumor immune escape and angiogenesis. Cancer Res 2012; 72: 2162-71.

72 Zhang, L., J.R. Conejo-Garcia, D. Katsaros, et al. Intratumoral T cells, recurrence, and survival in epithelial ovarian cancer. N Engl J Med 2003; 348: 203-13.

73 Hanahan, D. and R.A. Weinberg. The hallmarks of cancer. Cell 2000; 100: 57-70

74 Dirkx, A.E., M.G. Oude Egbrink, M.J. Kuijpers, et al. Tumor angiogenesis modulates leukocyte-vessel wall interactions in vivo by reducing endothelial adhesion molecule expression. Cancer Res 2003; 63: 2322-9.

75 Jain, R.K. Normalization of tumor vasculature: an emerging concept in antiangiogenic therapy. Science 2005; 307: 58-62.
76 Hamzah, J., M. Jugold, F. Kiessling, et al. Vascular normalization in Rgs5-deficient tumours promotes immune destruction. Nature 2008; 453: 410-4.

77 Bao, S., Q. Wu, S. Sathornsumetee, et al. Stem cell-like glioma cells promote tumor angiogenesis through vascular endothelial growth factor. Cancer Res 2006; 66: 7843-8.

78 Manning, E.A., J.G. Ullman, J.M. Leatherman, et al. A vascular endothelial growth factor receptor-2 inhibitor enhances antitumor immunity through an immune-based mechanism. Clin Cancer Res 2007; 13: 3951-9.

79 Shrimali, R.K., Z. Yu, M.R. Theoret, D. Chinnasamy, N.P. Restifo, and S.A. Rosenberg. Antiangiogenic agents can increase lymphocyte infiltration into tumor and enhance the effectiveness of adoptive immunotherapy of cancer. Cancer Res 2010; 70: 6171-80.

80 Huang, Y., J. Yuan, E. Righi, et al. Vascular normalizing doses of antiangiogenic treatment reprogram the immunosuppressive tumor microenvironment and enhance immunotherapy. Proc Natl Acad Sci U S A 2012; 109: 17561-6.

81 Pearlman, J.D., M.G. Hibberd, M.L. Chuang, et al. Magnetic resonance mapping demonstrates benefits of VEGF-induced myocardial angiogenesis. Nat Med 1995; 1: 1085-9.

82 Wada, J., H. Suzuki, R. Fuchino, et al. The contribution of vascular endothelial growth factor to the induction of regulatory T-cells in malignant effusions. Anticancer Res 2009; 29: 881-8.

83 Gupta, S., K. Joshi, J.D. Wig, and S.K. Arora. Intratumoral FOXP3 expression in infiltrating breast carcinoma: Its association with clinicopathologic parameters and angiogenesis. Acta Oncol 2007; 46: 792-7.

84 Giatromanolaki, A., G.J. Bates, M.I. Koukourakis, et al. The presence of tumor-infiltrating FOXP3 + lymphocytes correlates with intratumoral angiogenesis in endometrial cancer. Gynecol Oncol 2008; 110: 216-21.

85 Casares, N., L. Arribillaga, P. Sarobe, et al. CD4+/CD25+ regulatory cells inhibit activation of tumor-primed CD4 $+\mathrm{T}$ cells with IFNgamma-dependent antiangiogenic activity, as well as long-lasting tumor immunity elicited by peptide vaccination. J Immunol 2003; 171: 5931-9.

86 Zhan, H.L., X. Gao, X.F. Zhou, X.Y. Pu, and D.J. Wang. Presence of tumour-infiltrating FOXP3+ lymphocytes correlates with immature tumour angiogenesis in renal cell carcinomas. Asian Pac J Cancer Prev 2012; 13: 867-72.

87 Zhou, X., B. Li, H. Fan, and Z. Liu. Control of regulatory T cells and $T$ helper cells in human diseases: from bench to bedside. $J$ Mol Cell Biol 2013; 5: 210-1.

88 Schmitt, F., P. Govindaswamy, G. Suss-Fink, et al. Ruthenium porphyrin compounds for photodynamic therapy of cancer. J Med Chem 2008; 51: 1811-6.

89 Abe, F., I. Younos, S. Westphal, et al. Therapeutic activity of sunitinib for Her2/neu induced mammary cancer in FVB mice. Int Immunopharmacol 2010; 10: 140-5.

90 Adotevi, O., H. Pere, P. Ravel, et al. A decrease of regulatory T cells correlates with overall survival after sunitinib-based antiangiogenic therapy in metastatic renal cancer patients. J Immunother 2010; 33: 991-8.

91 Zaiss, M.M., K. Sarter, A. Hess, et al. Increased bone density and resistance to ovariectomy-induced bone loss in FoxP3-transgenic mice based on impaired osteoclast differentiation. Arthritis Rheum 2010; 62: 2328-38.

92 Mahnke, K., T. Bedke, and A.H. Enk. Regulatory conversation between antigen presenting cells and regulatory $T$ cells enhance immune suppression. Cell Immunol 2007; 250: 1-13.

93 Hilchey, S.P. and S.H. Bernstein. Use of CFSE to monitor ex vivo regulatory T-cell suppression of CD4 + and CD8 + T-cell proliferation within unseparated mononuclear cells from malignant and nonmalignant human lymph node biopsies. Immunol Invest 2007; 36: $629-48$

94 Kong, N., Q. Lan, M. Chen, et al. Induced T regulatory cells suppress osteoclastogenesis and bone erosion in collagen-induced arthritis better than natural T regulatory cells. Ann Rheum Dis 2012; 71: 1567-72. 
95 Pasparakis, M., I. Haase, and F.O. Nestle. Mechanisms regulating skin immunity and inflammation. Nat Rev Immunol 2014; 14: 289-301.

96 Gebhardt, T., L.M. Wakim, L. Eidsmo, P.C. Reading, W.R. Heath, and F.R. Carbone. Memory T cells in nonlymphoid tissue that provide enhanced local immunity during infection with herpes simplex virus. Nat Immunol 2009; 10: 524-30.

97 Rosenblum, M.D., I.K. Gratz, J.S. Paw, K. Lee, A. MarshakRothstein, and A.K. Abbas. Response to self antigen imprints regulatory memory in tissues. Nature 2011; 480: 538-42.

98 Graham, J.B., A. Da Costa, and J.M. Lund. Regulatory T cells shape the resident memory $T$ cell response to virus infection in the tissues. J Immunol 2013; 192: 683-90.

99 Sanchez Rodriguez, R, M.L. Pauli, I.M. Neuhaus, et al. Memory regulatory T cells reside in human skin. J Clin Invest 2014; 124: 1027-36.
100 Wiig, H., A. Schroder, W. Neuhofer, et al. Immune cells control skin lymphatic electrolyte homeostasis and blood pressure. J Clin Invest 2013; 123: 2803-15.

101 Petukhova, L., M. Duvic, M. Hordinsky, et al. Genome-wide association study in alopecia areata implicates both innate and adaptive immunity. Nature 2010; 466: 113-7.

(c) (1) (2) (2) This work is licensed under a Creative Commons Attribution-NonCommercial-ShareAlike 3.0 Unported License. The images or other third party material in this article are included in the article's Creative Commons license, unless indicated otherwise in the credit line; if the material is not included under the Creative Commons license, users will need to obtain permission from the license holder to reproduce the material. To view a copy of this license, visit http://creativecommons.org/licenses/by-nc-sa/3.0/ 